\title{
DIFERENTES GRAUS DE COMPACTACÃO E FORNECIMENTO DE FÓSFORO INFLUENCIANDO NO CRESCIMENTO DE PLANTAS DE MILHO (Zea mays L.) CULTIVADAS EM SOLOS DISTINTOS ${ }^{1}$
}

\author{
Different compaction degrees and phosphorus supply influencing the corn plants growth \\ (Zea mays $\mathbf{L}$.) cultivated in different soils
}

\author{
Gislene Aparecida dos Santos ${ }^{2}$, Moacir de Souza Dias Junior ${ }^{3}$, Paulo Tácito Gontijo Guimarães ${ }^{4}$, \\ Antonio Eduardo Furtini Neto 5
}

\section{RESUMO}

O crescimento e desenvolvimento das plantas podem ser limitados por impedimentos físicos e químicos que impedem o desenvolvimento radicular e restringe a absorção de nutrientes. Com a finalidade de avaliar o efeito de diferentes graus de compactação e doses de fósforo, na produção de matéria seca, teores de fósforo e fósforo acumulado na parte aérea do milho (Zea mays L.), um experimento foi conduzido em casa-de-vegetação, usando três solos, sendo um mais argiloso, Latossolo Vermelho Distrófico típico (LVd), um com textura média, Latossolo Vermelho-Amarelo Distrófico (LVAd) e um com textura arenosa, Neossolo Quartzarênico (RQ). Na montagem dos vasos, foi feita a calagem com calcário dolomítico e a adubação básica, sendo os graus de compactação obtidos mediante o ensaio de Proctor Normal. As maiores produções de MSPA, TPPA e PAPA foram obtidas nos graus de compactação 65 e $75 \%$ e na maior dose de fósforo aplicada. Em um mesmo grau de compactação a maior produção de matéria seca da parte aérea (MSPA), os maiores teores de fósforo (TPPA) e os maiores teores de fósforo acumulado na parte aérea (PAPA), ocorreram na maior dose de fósforo aplicado. O fósforo aplicado funcionou como um fator de aliviamento da compactação do solo, resultando em uma maior produção para um mesmo grau de compactação.

Termos de indexação: Graus de compactação, Proctor normal, Fósforo e Milho.

\begin{abstract}
The growth and development of the plants can be limited by physical and chemical impediments that limit roots development and restricts nutrients absorption. With the purpose of evaluating the effect of different degrees of compaction and phosphorus doses, in the production of dry matter, level of phosphorus and accumulated phosphorus in the aerial part of the corn (Zea mays L.), an experiment was set up in a green house conditions, using three soils, being a clay one, distrofic Red Latosol, one with loamy texture, distrofic Red Yellow Latosol and one with sandy texture, Quartzarenic Neosol. In the assembly of the vases, liming was made with dolomitic limestone and the basic manuring, being the compaction degrees obtained through the Normal Proctor test. The largest productions of MSPA, TPPA and PAPA were obtained in the compaction degrees of 65 and $75 \%$ and in the largest applied phosphorus dose. In the same compaction degree the largest production of dry matter of the aerial part (MSPA), the largest phosphorus level (TPPA) and the largest level of accumulated phosphorus in the aerial part (PAPA), occurred in the largest dose of applied phosphorus. The applied phosphorus worked as an alleviating factor of the soil compaction, resulting in a larger production for a same compaction degree.
\end{abstract}

Index terms: Degree of Compaction, Normal Proctor test, phosphorus and Corn.

(Recebido para publicação em 5 de março de 2004 e aprovado 25 de maio de 2005

\section{INTRODUÇÃO}

O crescimento e desenvolvimento das plantas são dependentes de vários fatores. A produção pode ser limitada por fatores físicos e químicos que impedem o desenvolvimento radicular e restringe a absorção de nutrientes (CAMARGO \& ALLEONI, 1997).

O principal processo de movimento do fósforo no solo é por difusão. Como o movimento do fósforo por difusão até as raízes das plantas é restrito, esse processo é geralmente considerado como um fator limitante na sua absorção (GRANT et al., 2001). Devido à baixa mobilidade do fósforo a sua absorção pode ficar ainda mais comprometida em solos compactados, devido ao fato da resistência mecânica do solo reduzir a habilidade das raízes em absorver o fósforo, como é o caso do milho (FOLONI et al., 2003; SHIERLAW \& ALSTON, 1984).

\footnotetext{
${ }_{1}$ Parte da Dissertação de Mestrado do primeiro autor, apresentada ao DCS/UFLA. Projeto financiado pela EPAMIG/UFLA

${ }^{2}$ Engenheira Agrícola, MS, Departamento de Ciência do Solo, Universidade Federal de Lavras/UFLA - Caixa Postal 3037 - $37200-000$ - Lavras, MG Bolsista do CNPq - gisant@ufla.br

${ }^{3}$ Professor Adjunto do Departamento de Ciência do Solo - Universidade Federal de Lavras/UFLA - Bolsista do CNPq.

${ }^{4}$ Pesquisador Doutor, CTSM/EPAMIG - Universidade Federal de Lavras/UFLA.

${ }^{5}$ Professor Adjunto do Departamento de Ciência do Solo - Universidade Federal de Lavras/UFLA - Bolsista do CNPq.
} 
A compactação altera as propriedades físicas do solo podendo causar redução no comprimento do sistema radicular e aumento no seu diâmetro (VEPRASKAS, 1994), como consequiência do aumento da resistência mecânica que as raízes têm que vencer para crescerem. Estas alterações das propriedades físicas do solo podem ocorrer mais facilmente devido ao feito dispersivo do fósforo nos colóides do solo (HINGSTON et al., 1972; SAWHNEY, 1974; SPOSITO, 1989), tornando-o mais susceptível a compactação, como foi observado por Silva (1998).

Apesar da compactação do solo poder promover uma concentração superficial das raízes (BARBER, 1995), a aplicação de nutrientes, como fósforo e o nitrogênio, em linhas, faixas ou a lanço na superfície do solo, tem sido usado com a finalidade de melhorar o estado nutricional das plantas e o desenvolvimento das culturas, promovendo um maior crescimento das raízes nestas condições (ANGHINONI, 1992; ANGHINONI \& BARBER, 1980; BORKERT \& BARBER, 1985; KLEPKER \& ANGHINONI, 1993, 1995). Assim, estudos que visam quantificar o efeito do fósforo como um fator de aliviamento da compactação, é interessante, pois minimizaria o efeito da compactação na produtividade.

Os ensaios mais utilizados para estudar a compactação do solo em laboratório têm sido o ensaio de Proctor Normal e o ensaio de compressão uniaxial. Estes ensaios são muito utilizados na Engenharia Civil. Entretanto, pesquisas têm sido conduzidas para fins agrícolas usando estes ensaios (BARZEAGAR \& ASSODAR, 2000; EKWUE \& STONE, 1997; FIGUEIREDO \& DIAS JUNIOR, 1997; MIRANDA, 2001; OLIVEIRA et al., 2003; SILVA, 1999), com o objetivo de verificar a influência da umidade (DIAS JUNIOR \& PIERCE, 1996), da textura (PACHECO \& DIAS JUNIOR, 1990), da matéria orgânica (MORAES, 1988; TORRES et al., 1993), etc, na susceptibilidade a compactação dos solos.

Vários estudos sobre compactação do solo têm utilizado a densidade do solo como indicador do nível de compactação (BORGES et al., 1999; DÍAZ-ZORITA, 2000; KRZIC et al., 2000; QUEIROZ-VOLTAN et al., 2000). Entretanto, outros estudos utilizaram o grau de compactação como indicador do nível de compactação (CORRÊA et al., 1998; DIAS JUNIOR \& ESTANISLAU, 1999; MIRANDA \& DIAS JUNIOR 1998), devido ao fato deste permitir uma normalização dos valores de densidade do solo iniciais através da densidade do solo máxima obtida no ensaio de Proctor Normal.

Neste contexto, objetivou-se com este trabalho avaliar o crescimento de plantas do milho (Zea mays L.), em função de diferentes graus de compactação e doses de fósforo em três classes de solo.

\section{MATERIALEMÉTODOS}

Para avaliar o efeito do grau de compactação e doses de fósforo no crescimento do milho (Zea mays L.), foram coletadas amostras deformadas e indeformadas na profundidade de $0-20 \mathrm{~cm}$ em um Latossolo Vermelho Distrófico típico (LVd), textura argilosa; em um Latossolo Vermelho-Amarelo Distrófico (LVAd), textura média, e em um Neossolo Quartzarênico (RQ), textura arenosa.

As amostras indeformadas foram utilizadas na determinação da densidade do solo (Ds) e as deformadas nas determinações físicas e químicas e na montagem dos vasos. O material do solo foi caracterizado química e fisicamente conforme Embrapa (1997) (Quadros 1 e 2).

Na realização dos ensaios de Proctor Normal foram utilizados $5 \mathrm{~kg}$ de solo provenientes das amostras deformadas, os quais foram previamente secos ao ar e peneirados em peneira $4,76 \mathrm{~mm}$. O ensaio de Proctor Normal foi realizado com o reuso do material de solo e o corpo de prova consistiu de três camadas, que foram submetidas a 25 golpes por camada com um soquete de $2,5 \mathrm{~kg}$ e cada camada ocupou 1/3 do volume do cilindro. Após, compactado o corpo de prova, o mesmo foi extraído, coletando uma amostra do seu centro para a determinação da umidade e da densidade do solo.

Após a obtenção dos 5 valores de densidade do solo (Ds) e de umidade gravimétrica (U), plotaram-se os resultados em um gráfico, Ds $=\mathrm{f}(\mathrm{U})$ (STANCATI et al., 1981). Ajustou-se, a seguir, um polinômio do segundo grau, obtendo-se, então, a curva de compactação (Figura 1). A umidade ótima ou crítica de compactação foi obtida pela expressão: $\mathrm{U}_{\text {ot. }}=(-\mathrm{b} / 2 \mathrm{a})$ e a densidade máxima $\mathrm{Ds}_{\text {máx }}=(-\mathrm{D} /$ 4a) (MIRANDA \& DIAS JUNIOR, 1998) (Quadro 3). Os Graus de Compactação foram obtidos utilizando-se a expressão:

\section{G.C. $=$ Ds $($ campo)/Ds (máxima de laboratório $)$}

A escolha das densidades do solo para cada classe de solo foi feita de tal modo que houvesse quatro graus de compactação situados no ramo seco da Curvas de compactação de cada classe de solo.

Os experimentos foram realizados utilizando-se os graus de compactação e as densidades do solo máxima conforme Quadro 3. 
QUADRO 1 - Caracterização física e química de um Neossolo Quartzarênico (RQ), textura arenosa; Latossolo Vermelho-Amarelo Distrófico (LVAd), textura média e de um Latossolo Vermelho Distrófico (LVAd), textura argilosa.

\begin{tabular}{|c|c|c|c|}
\hline & $\mathbf{R Q}$ & LVAd & LVd \\
\hline pH em água $(1: 2,5)$ & 4,8 & 5,1 & 5,0 \\
\hline P - Mehlich $1\left(\mathrm{mg} \mathrm{dm}^{-3}\right)$ & 10,0 & 1,0 & 1,5 \\
\hline $\mathrm{K}$ - Mehlich $1\left(\mathrm{mg} \mathrm{dm}^{-3}\right)$ & 24,0 & 31,0 & 39,0 \\
\hline $\mathrm{Ca}\left(\mathrm{cmol}_{\mathrm{c}} \mathrm{dm}^{-3}\right)$ & 0,4 & 0,4 & 0,5 \\
\hline $\mathrm{Mg}\left(\mathrm{cmol}_{\mathrm{c}} \mathrm{dm}^{-3}\right)$ & 0,1 & 0,1 & 0,2 \\
\hline $\mathrm{Al}\left(\mathrm{cmol}_{\mathrm{c}} \mathrm{dm}^{-3}\right)$ & 1,1 & 0,8 & 0,9 \\
\hline $\mathrm{t}(\mathrm{CTC}$ efetiva $)\left(\mathrm{cmol}_{\mathrm{c}} \mathrm{dm}^{-3}\right)$ & 1,7 & 1,4 & 1,7 \\
\hline T $\left(\right.$ CTC a pH 7,0) $\left(\mathrm{cmol}_{\mathrm{c}} \mathrm{dm}^{-3}\right)$ & 6,2 & 5,6 & 7,1 \\
\hline $\mathrm{Zn}\left(\mathrm{mg} \mathrm{dm}^{-3}\right)$ & 0,5 & 3,7 & 0,4 \\
\hline $\mathrm{V}(\%)$ & 10,1 & 10,4 & 11,3 \\
\hline Matéria Orgânica (dag dm ${ }^{-3}$ ) & 2,2 & 0,1 & 2,7 \\
\hline Densidade do solo $\left(\mathrm{Mg} \mathrm{m}^{-3}\right)$ & 1,25 & 1,34 & 1,28 \\
\hline Densidade de partículas $\left(\mathrm{Mg} \mathrm{m}^{-3}\right)$ & 2,68 & 2,63 & 2,81 \\
\hline
\end{tabular}

QUADRO 2 - Caracterização física do Latossolo Vermelho distrófico típico (LVd), textura argilosa; do Latossolo-Vermelho Amarelo distrófico (LVAd), textura média, e do Neossolo Quartzarênico (RQ), textura arenosa.

\begin{tabular}{|c|c|c|c|c|c|c|c|c|}
\hline \multirow[t]{2}{*}{ Solo } & \multicolumn{5}{|c|}{ Areia } & \multirow{2}{*}{$\begin{array}{l}\text { Areia } \\
\text { Total }\end{array}$} & \multirow[t]{2}{*}{ Argila } & \multirow[t]{2}{*}{ Silte } \\
\hline & AMG & $\mathrm{AG}$ & AM & $\mathrm{AF}$ & AMF & & & \\
\hline \multicolumn{9}{|c|}{-- ------------------------------------ $\mathrm{g} \mathrm{kg}^{-1}$------------------------------------------- } \\
\hline RQ & 8,0 & 90,0 & 376,0 & 384,0 & 69,0 & 927,0 & 49,0 & 24,0 \\
\hline LVAd & 26,0 & 130,0 & 160,0 & 179,0 & 32,0 & 527,0 & 326,0 & 147,0 \\
\hline $\mathrm{LVd}$ & 70,0 & 130,0 & 102,0 & 84,0 & 28,0 & 414,0 & 356,0 & 230,0 \\
\hline
\end{tabular}

Em que: $\mathrm{AMG}=$ Areia Muito Grossa (2-1mm); $\mathrm{AG}=$ Areia Grossa (1-0,5 mm); AM = Areia Média (0,5-0,25 mm); $\mathrm{AF}=$ Areia Fina $(0,25-0,10 \mathrm{~mm}) ; \mathrm{AMF}=$ Areia Muito Fina $(0,10-0,05 \mathrm{~mm})-$ diâmetro das partículas segundo classificação Americana - USDA. 


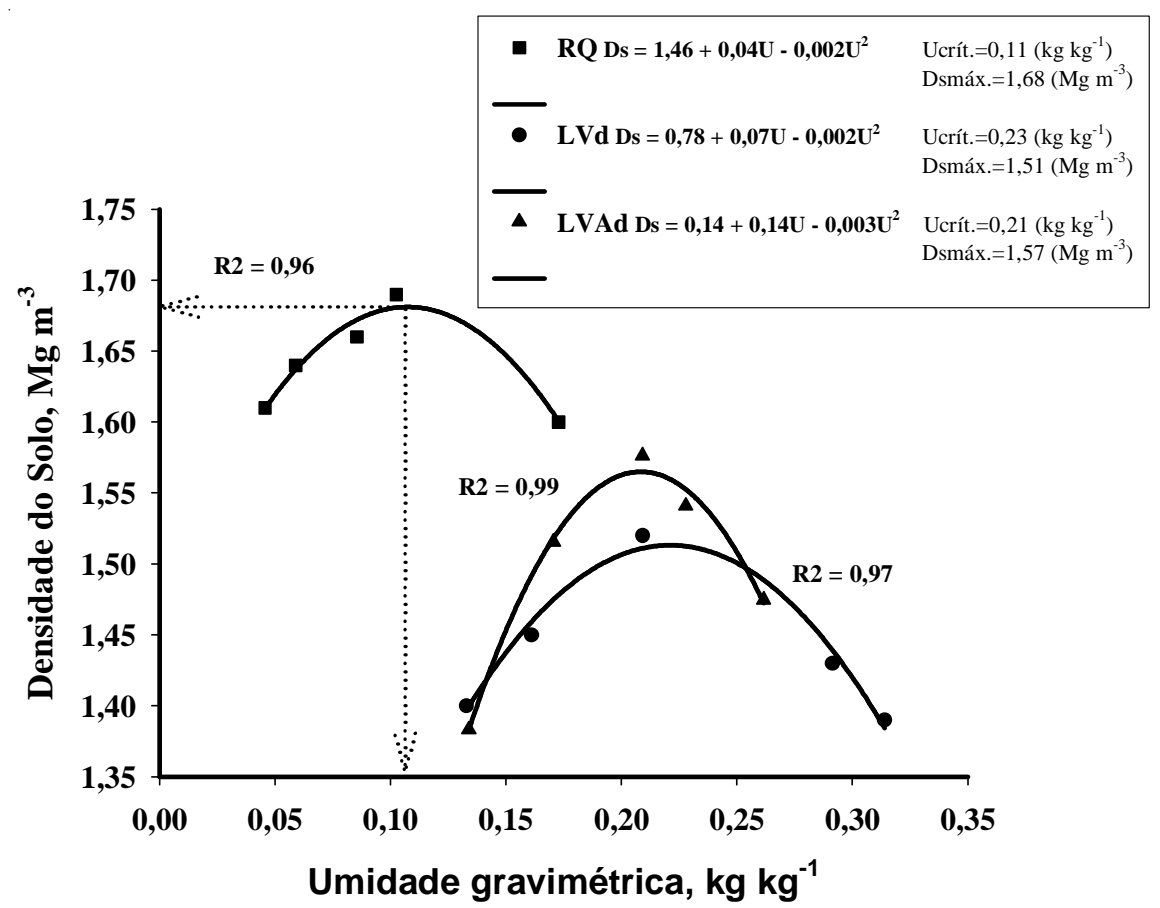

FIGURA 1 - Curvas de compactação do Latossolo Vermelho Distrófico típico (LVd), textura argilosa; do Latossolo Vermelho-Amarelo Distrófico (LVAd), textura média, e do Neossolo Quartzarênico (RQ), textura arenosa.

QUADRO 3 - Graus de compactação, densidades do solo, densidades do solo máxima e umidades críticas de compactação do Neossolo Quartzarênico (RQ), textura arenosa, do Latossolo Vermelho-Amarelo Distrófico (LVAd), textura média, e do Latossolo Vermelho Distrófico típico (LVd), textura argilosa.

\begin{tabular}{|c|c|c|c|c|}
\hline Solo & GC (\%) & $\begin{array}{c}\text { Ds }_{\text {vaso }} \\
\left(\mathrm{Mg} \mathrm{m}^{-3}\right)\end{array}$ & $\begin{array}{l}D_{\text {máx. }}{ }^{(1)} \\
\left(\mathbf{M g ~ m}^{-3}\right)\end{array}$ & $\begin{array}{c}\mathrm{U}_{\text {critit. }}{ }^{(1)} \\
\left(\mathrm{kg} \mathrm{kg}^{-1}\right)\end{array}$ \\
\hline & 72 & 1,22 & \multirow{4}{*}{1,68} & \multirow{4}{*}{0,11} \\
\hline \multirow[t]{4}{*}{ RQ } & 75 & 1,27 & & \\
\hline & 85 & 1,44 & & \\
\hline & 95 & 1,60 & & \\
\hline & 65 & 1,02 & \multirow{4}{*}{1,57} & \multirow{4}{*}{0,21} \\
\hline \multirow[t]{4}{*}{ LVAd } & 75 & 1,18 & & \\
\hline & 85 & 1,33 & & \\
\hline & 95 & 1,49 & & \\
\hline & 65 & 0,98 & \multirow{4}{*}{1,51} & \multirow{4}{*}{0,23} \\
\hline \multirow[t]{3}{*}{$\mathrm{LVd}$} & 75 & 1,13 & & \\
\hline & 85 & 1,28 & & \\
\hline & 95 & 1,43 & & \\
\hline
\end{tabular}

Em que: $\mathrm{GC}=$ Grau de compactação; $\mathrm{Ds}_{\text {vaso }}=$ Densidade do solo do vaso; $\mathrm{Ds}_{\text {máx. }}=$ Densidade do solo máxima; $\mathrm{U}_{\text {crít. }}=$ Umidade crítica de compactação. 1= média de 4 repetições. 
Na montagem dos vasos, usou-se $1,8 \mathrm{dm}^{3}$ de solo peneirado em peneira de malha $4,76 \mathrm{~mm}$. A calagem foi feita com calcário dolomítico com PRNT $=100 \%$, para o V\% $=60 \%$, e a adubação básica da seguinte maneira: $S=40$ $\mathrm{mg} \mathrm{dm}{ }^{-3}, \mathrm{~K}=150 \mathrm{mg} \mathrm{dm}^{-3}, \mathrm{~N}=100 \mathrm{mg} \mathrm{dm}^{-3}, \mathrm{~B}=0,8 \mathrm{mg} \mathrm{dm}^{-3}$, $\mathrm{Cu}=1,3 \mathrm{mg} \mathrm{dm}^{-3}, \mathrm{Mn}=3,6 \mathrm{mg} \mathrm{dm}^{-3}, \mathrm{Fe}=1,5 \mathrm{mg} \mathrm{dm}^{-3}$, $\mathrm{Mo}=0,15 \mathrm{mg} \mathrm{dm}^{-3} \mathrm{e} Z \mathrm{n} 5,0 \mathrm{mg} \mathrm{dm}^{-3}$. Utilizaram-se soluções contendo nutrientes, oriundas das seguintes fontes: $\mathrm{K}_{2} \mathrm{SO}_{4}, \mathrm{KCl}, \mathrm{NH}_{4} \mathrm{NO}_{3}, \mathrm{H}_{3} \mathrm{BO}_{3}, \mathrm{CuSO}_{4} 5 \mathrm{H}_{2} \mathrm{O}, \mathrm{MnSO}_{4} \mathrm{H}_{2} \mathrm{O}$, $\mathrm{Fe}_{\text {EDTA }}, \mathrm{H}_{2} \mathrm{MoO}_{4} \mathrm{H}_{2} \mathrm{O}, \mathrm{Zn} \mathrm{SO}_{4} 7 \mathrm{H}_{2} \mathrm{O}$. A fertilização com o fósforo para $\mathrm{LVd}$ e LVAd, com doses correspondentes a 0, 100, $200 \mathrm{e} 400 \mathrm{mg} \mathrm{dm}^{-3}$; e para o RQ, as doses foram 0 , 50,100 e $200 \mathrm{mg} \mathrm{dm}^{-3}$. Na fertilização com o fósforo, foram utilizadas as fontes $\mathrm{H}_{3} \mathrm{PO}_{4}$ e $\mathrm{NH}_{4} \mathrm{H}_{2} \mathrm{PO}_{4}$. Utilizou-se solução nutritiva para a fertilização e aplicação por meio de pipetagem para a adubação básica e aplicação das doses de fósforo. Após a fertilização, os solos permaneceram incubados por 22 dias com uma umidade aproximadamente igual à capacidade de campo. Após a incubação, os solos foram secos ao ar novamente e peneirados em malha de $4,76 \mathrm{~mm}$.

Na montagem dos vasos usou-se um tubo de PVC com $15 \mathrm{~cm}$ de diâmetro e $10 \mathrm{~cm}$ de altura. A compactação dos vasos foi feita com o soquete do ensaio de Proctor Normal e, para que não houvesse deformação do anel no momento da compactação, foi necessária a utilização de uma braçadeira confeccionada em latão, que revestia o tubo de PVC.

O plantio do milho foi feito diretamente nos vasos de cultivo, inicialmente, com 6 sementes e após o desbaste, foram cultivadas 3 plantas até o final do experimento. Durante o período experimental, a umidade do solo nos anéis foi mantida em $70 \%$ do VTP, mediante pesagens diárias e repondo-se o volume evapotranspirado com água desmineralizada.

Após 35 dias de cultivo, as plantas foram cortadas rente ao solo e o material vegetal separado em parte aérea e sistema radicular, procedendo as seguintes avaliações: matéria seca da parte aérea; teor e acúmulo de fósforo na parte aérea.

O material vegetal seco em estufa com circulação de ar a $70^{\circ} \mathrm{C}$ até peso constante, foi submetido à pesagem $\mathrm{e}$ determinação da matéria seca. Os teores de fósforo na matéria seca da parte aérea foram determinados no extrato obtido pela digestão nitro-perclórica (SARRUGE \& HAAG, 1974).

O delineamento experimental usado foi o inteiramente casualizado, arranjado em esquema fatorial 4 x 4 x 3 , sendo quatro graus de compactação, quatro doses de fósforo e três repetições, para cada solo.

Para a estatística foi utilizada a análise de regressão quadrática e para as figuras o software Jadel Scientific Sigma Plot; com significância ao nível de 5\% em função dos Graus de Compactação e Doses de Fósforo.

\section{RESULTADOSE DISCUSSÃO}

\section{Neossolo Quartzarênico (RQ)}

A produção de matéria seca da parte aérea (MSPA), o Teor de Fósforo na Parte Aérea (TPPA) e o Fósforo Acumulado na Parte Aérea (PAPA), nas doses 100 e $200 \mathrm{mg} \mathrm{dm}^{-3}$, foram influenciadas significativamente pelo grau de compactação (Figura 2 e Quadro 4). O grau de compactação não influenciou significativamente na MSPA, TPPA e PAPA quando não se aplicou fósforo e na dose de $50 \mathrm{mg} \mathrm{dm}^{-3}$. Em um mesmo grau de compactação a MSPA, TPPA e PAPA foram maiores quando maiores doses de fósforo foram aplicadas (Figura 2 e Quadro 4), indicando um aliviamento dos efeitos adversos da compactação pela aplicação de maiores doses de fósforo.

As maiores produções de MSPA foram 5,36 e 5,27 g vaso $^{-1}$ obtidas na dose de $200 \mathrm{mg} \mathrm{dm}^{-3}$ de fósforo para os graus de compactação (GC) 75\% e 72\%, respectivamente. $\mathrm{O}$ aumento da produção nesses graus de compactação pode ter sido devido a maior disponibilidade de fósforo (BORGES, 1995), promovido pelo maior contato solo/raiz, o que pode ter favorecido o processo de difusão, o que não ocorreu, nos graus de compactação mais altos (85 e 95\%).

À medida que o grau de compactação aumenta para a dose $100 \mathrm{mg} \mathrm{dm}^{-3}$, ocorre uma redução linear do TPPA e PAPA. Na dose $200 \mathrm{mg} \mathrm{dm}^{-3}$, a equação de regressão que correlaciona o TPPA e o PAPA com o grau de compactação seguiram uma equação do segundo grau, sendo o TPPA máximo $\left(2,59 \mathrm{~g} \mathrm{~kg}^{-1}\right)$ ocorreu no $\mathrm{GC}=81 \%$, e o PAPA máximo $\left(12,82 \mathrm{mg} \mathrm{kg}^{-1}\right)$ ocorreu no $\mathrm{GC}=76 \%$.

Ciênc. agrotec., Lavras, v. 29, n. 4, p. 740-752, jul./ago., 2005 


\section{NEOSSOLO QUARTZARENICO}

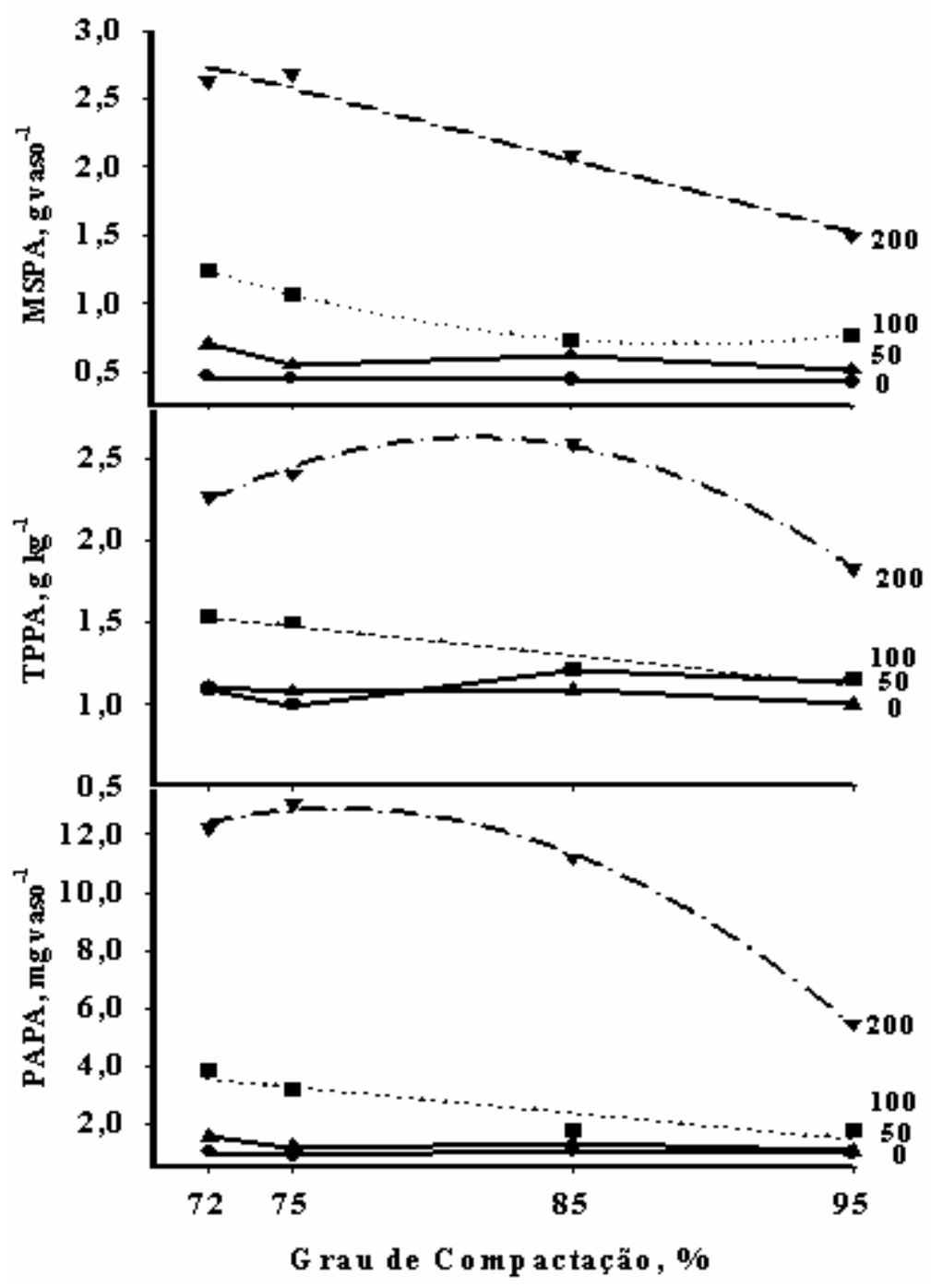

FIGURA 2 - Matéria Seca da Parte Aérea (MSPA), Teor de Fósforo na Parte Aérea (TPPA) e Fósforo Acumulado na Parte Aérea (PAPA) de plantas de milho cultivadas em um Neossolo Quartzarênico. 
QUADRO 4 - Equações significativas ao nível de 5\% (Neossolo Quartzarênico):

\begin{tabular}{|c|c|c|c|c|c|c|}
\hline \multirow{2}{*}{$\begin{array}{c}\text { Grau de } \\
\text { compactação } \\
(\%)\end{array}$} & \multicolumn{4}{|c|}{$\begin{array}{l}\text { Dose de Fósforo } \\
\qquad\left(\mathrm{mg} \mathrm{dm}^{-3}\right)\end{array}$} & \multicolumn{2}{|l|}{$\begin{array}{c}\text { Equações da Regressão } \\
\text { Significancia à } 5 \%\end{array}$} \\
\hline & $\mathbf{0}$ & 100 & 200 & 400 & & $\mathbf{R}^{2}$ \\
\hline \multicolumn{7}{|c|}{ Matéria seca da Parte Aérea - MSPA $\left(\right.$ g vaso $\left.^{-1}\right)$} \\
\hline 72 & 0,94 & 1,42 & 2,49 & 5,27 & $\mathrm{MSPA}_{(0)}=\mathrm{NS}$ & \\
\hline 75 & 0,90 & 1,11 & 2,13 & 5,36 & $\operatorname{MSPA}_{(50)}=\mathrm{NS}$ & \\
\hline 85 & 0,89 & 1,25 & 1,48 & 4,18 & MSPA $_{(100)}=30,4546-0,6551 \mathrm{GC}+0,0057 \mathrm{GC}^{2}$ & 0,99 \\
\hline 95 & 0,86 & 1,04 & 1,54 & 2,99 & $\operatorname{MSPA}_{(200)}=13,4546-0,1052 \mathrm{GC}$ & 0,98 \\
\hline \multicolumn{7}{|c|}{ Teor de Fósforo na Pate Aérea - TPPA $\left(\mathrm{g} \mathrm{kg}^{-1}\right)$} \\
\hline 72 & 1,09 & 1,10 & 1,54 & 2,27 & $\mathrm{TPPA}_{(0)}=\mathrm{NS}$ & \\
\hline 75 & 0,99 & 1,08 & 1,50 & 2,41 & $\mathrm{TPPA}_{(50)}=\mathrm{NS}$ & \\
\hline 85 & 1,21 & 1,09 & 1,21 & 2,59 & $\mathrm{TPPA}_{(100)}=2,813-0,018 \mathrm{GC}$ & 0,91 \\
\hline 95 & 1,14 & 1,00 & 1,16 & 1,83 & TPPA $_{(200)}=-25,8987+0,701 \mathrm{GC}-0,0043 \mathrm{GC}^{2}$ & 0,99 \\
\hline \multicolumn{7}{|c|}{ Fósforo Acumulado na Parte Aérea - PAPA $\left(\mathrm{mg} \mathrm{vaso}^{-1}\right)$} \\
\hline 72 & 1,02 & 1,56 & 3,86 & 12,23 & PAPA $_{(0)}=\mathrm{NS}$ & \\
\hline 75 & 0,89 & 1,19 & 3,19 & 13,09 & $\mathrm{PAPA}_{(50)}=\mathrm{NS}$ & \\
\hline 85 & 1,08 & 1,35 & 1,79 & 11,19 & PAPA $_{(100)}=10,041-0,0903 \mathrm{GC}$ & 0,83 \\
\hline 95 & 0,98 & 1,04 & 1,80 & 5,46 & $\operatorname{PAPA}_{(200)}=-115,8305+3,3633 \mathrm{GC}-0,0219 \mathrm{GC}^{2}$ & 0,99 \\
\hline
\end{tabular}

\section{Latossolo Vermelho-Amarelo (LVAd)}

A produção de matéria seca da parte aérea (MSPA), o Teor de Fósforo na Parte Aérea (TPPA) exceto na dose $100 \mathrm{~g} \mathrm{~kg}^{-1}$, e o Fósforo Acumulado na Parte Aérea (PAPA), em todas as doses de fósforo aplicado, foram influenciadas significativamente pelo grau de compactação (Figura 3 e Quadro 5). Em um mesmo grau de compactação a MSPA, TPPA e PAPA foram maiores quando maiores doses de fósforo foram aplicadas (Figura 3 e Quadro 5), indicando um aliviamento dos efeitos adversos da compactação pela aplicação de maiores doses de fósforo.

À medida que o grau de compactação aumenta para a dose $100 \mathrm{mg} \mathrm{dm}^{-3}$, ocorre uma redução linear da MSPA e PAPA. Nas doses 0,200 e $400 \mathrm{mg} \mathrm{dm}^{-3}$, as equações de regressão que correlacionam a MSPA, TPPA e PAPA com o grau de compactação, seguiram uma equação do segundo grau, sendo que na dose $400 \mathrm{mg} \mathrm{dm}^{-3}$, obteve-se a MSPA máxima $\left(6,02 \mathrm{~g} \mathrm{~kg}^{-1}\right)$ que ocorreu no $\mathrm{GC}=77 \%$, o TPPA máximo $\left(2,80 \mathrm{~g} \mathrm{~kg}^{-1}\right)$ que ocorreu no $\mathrm{GC}=65 \%$, e o PAPA máximo $\left(15,62 \mathrm{mg} \mathrm{kg}^{-1}\right)$ que ocorreu no $\mathrm{GC}=75 \%$. Comportamento semelhante foi observado por Santos et al. (1998), quando cultivaram plantas de milho em um Latossolo Vermelho-Amarelo. Estes resultados corroboram com Corrêa et al. (1998) que avaliando o efeito da compactação na parte aérea do cafeeiro, concluíram que o alto grau de compactação influenciou na concentração de macro e micronutrientes, sendo que $\mathrm{P}$ e o $\mathrm{S}$ sofreram redução em todos os Latossolos em que as plantas foram cultivadas. Os menores valores de MSPA, TPPA e PAPA foram obtidos onde não ocorreu a aplicação de fósforo e para o $\mathrm{GC}=95 \%$. 


\section{LATOSSOLO VERMELHO-AMARELO DISTRÓFICO}

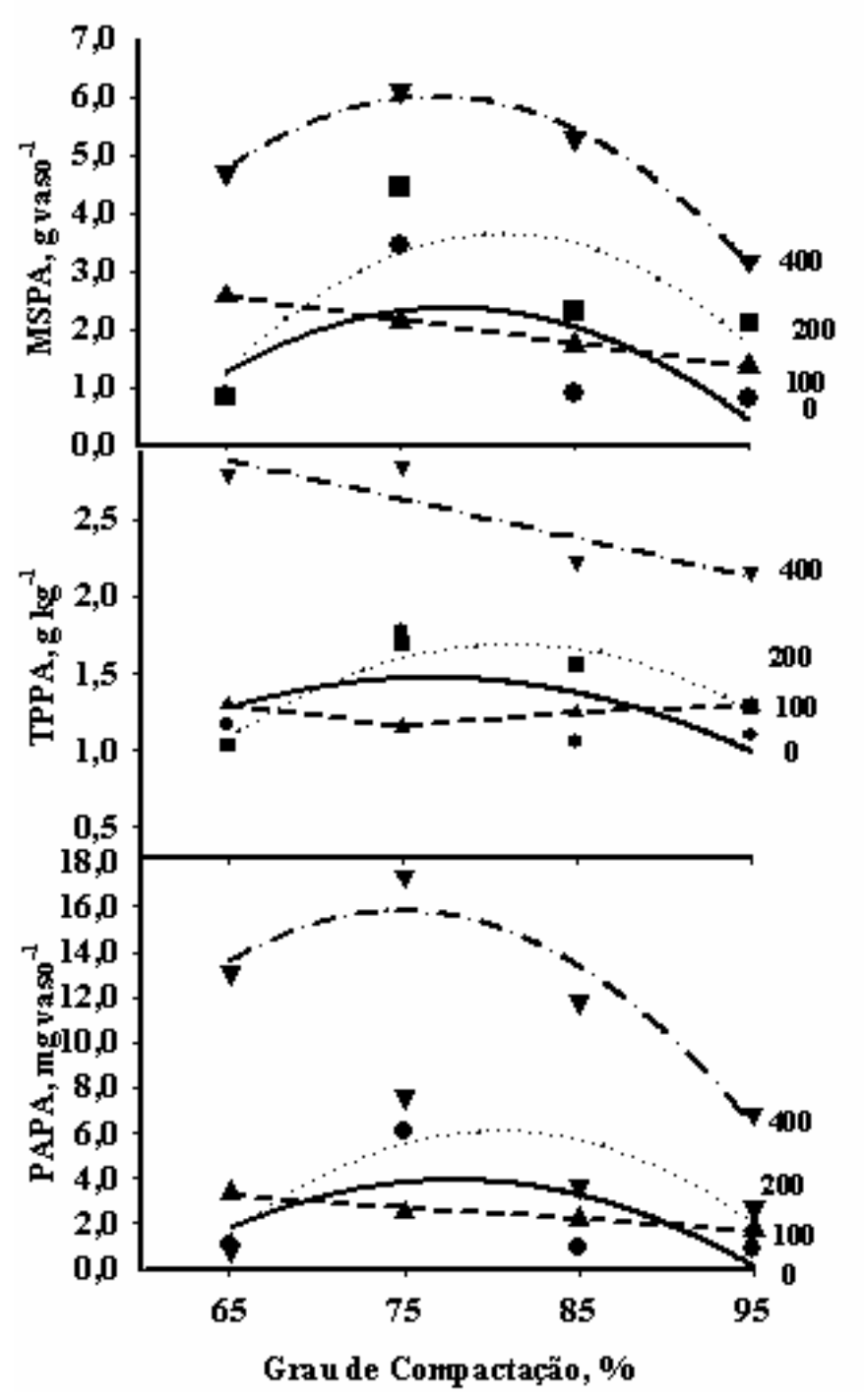

FIGURA 3 - Matéria Seca da Parte Aérea (MSPA), Teor de Fósforo na Parte Aérea (TPPA) e Fósforo Acumulado na Parte Aérea (PAPA) de plantas de milho cultivadas em um Latossolo Vermelho-Amarelo Distrófico. 
QUADRO 5 - Equações significativas ao nível de 5\% (Latossolo Vermelho-Amarelo Distrófico):

\begin{tabular}{|c|c|c|c|c|c|c|}
\hline \multirow{2}{*}{$\begin{array}{c}\text { Grau de } \\
\text { compactação } \\
(\%)\end{array}$} & \multicolumn{4}{|c|}{$\begin{array}{l}\text { Dose de Fósforo } \\
\left(\mathrm{mg} \mathrm{dm}^{-3}\right)\end{array}$} & \multicolumn{2}{|l|}{$\begin{array}{l}\text { Equações da Regressão } \\
\text { Significancia à } 5 \%\end{array}$} \\
\hline & $\mathbf{0}$ & 100 & 200 & 400 & & $\mathbf{R}^{2}$ \\
\hline \multicolumn{7}{|c|}{ Matéria seca da Parte Aérea - MSPA $\left(\right.$ g vaso $\left.^{-1}\right)$} \\
\hline 65 & 0,88 & 2,58 & 0,86 & 4,68 & $\operatorname{MSPA}_{(0)}=-37,729+1,037 \mathrm{GC}-0,007 \mathrm{GC}^{2}$ & 0,43 \\
\hline 75 & 3,44 & 2,16 & 4,46 & 6,11 & $\operatorname{MSPA}_{(\mathbf{1 0 0})}=5,219-0,041 \mathrm{GC}$ & 0,99 \\
\hline 85 & 0,90 & 1,75 & 2,34 & 5,29 & $\operatorname{MSPA}_{(200)}=-58,674+1,541 \mathrm{GC}-0,009 \mathrm{GC}^{2}$ & 0,56 \\
\hline 95 & 0,82 & 1,36 & 2,13 & 3,18 & $\operatorname{MSPA}_{(400)}=-46,464+1,363 \mathrm{GC}-0,009 \mathrm{GC}^{2}$ & 0,99 \\
\hline \multicolumn{7}{|c|}{ Teor de Fósforo na Pate Aérea - TPPA $\left(\mathrm{g} \mathrm{kg}^{-1}\right)$} \\
\hline 65 & 1,17 & 1,30 & 1,04 & 2,80 & $\operatorname{MSPA}_{(0)}=-6,920+0,219 \mathrm{GC}-0,001 \mathrm{GC}^{2}$ & 0,36 \\
\hline 75 & 1,78 & 1,16 & 1,70 & 2,85 & $\operatorname{MSPA}_{(\mathbf{1 0 0})}=\mathrm{NS}$ & \\
\hline 85 & 1,06 & 1,25 & 1,56 & 2,23 & $\operatorname{MSPA}_{(200)}=-13,678+0,378 \mathrm{GC}-0,002 \mathrm{GC}^{2}$ & 0,91 \\
\hline 95 & 1,10 & 1,29 & 1,29 & 2,16 & $\operatorname{MSPA}_{(400)}=4,542-0,0254 \mathrm{GC}$ & 0,81 \\
\hline \multicolumn{7}{|c|}{ Fósforo Acumulado na Parte Aérea - PAPA $\left(\mathrm{mg} \mathrm{vaso}^{-1}\right)$} \\
\hline 65 & 1,05 & 3,36 & 0,89 & 13,11 & $\operatorname{MSPA}_{(0)}=-73,585+1,992 \mathrm{GC}-0,0128 \mathrm{GC}^{2}$ & 0,41 \\
\hline 75 & 6,11 & 2,50 & 7,58 & 17,36 & $\operatorname{MSPA}_{(\mathbf{1 0 0})}=6,5240-0,051 \mathrm{GC}$ & 0,95 \\
\hline 85 & 0,96 & 2,22 & 3,67 & 11,82 & $\operatorname{MSPA}_{(\mathbf{2 0 0})}=-117,266+3,0681 \mathrm{GC}-0,019 \mathrm{GC}^{2}$ & 0,61 \\
\hline 95 & 0,90 & 1,76 & 2,73 & 6,88 & $\operatorname{MSPA}_{(400)}=-112,492+3,434 \mathrm{GC}-0,023 \mathrm{GC}^{2}$ & 0,90 \\
\hline
\end{tabular}

\section{Latossolo Vermelho Distrófico Típico (LVd)}

A produção de matéria seca da parte aérea (MSPA), nas dose 100,200 e $400 \mathrm{mg} \mathrm{dm}^{-3} \mathrm{e}$ o Fósforo Acumulado na Parte Aérea (PAPA), nas dose $200 \mathrm{e} 4000 \mathrm{mg} \mathrm{dm}^{-3}$, foram influenciadas significativamente pelo grau de compactação (Figura 4 e Quadro 6). O Teor de Fósforo na Parte Aérea (TPPA) não foi influenciado pelo grau de compactação. Em um mesmo grau de compactação a MSPAe PAPA foram maiores quando maiores doses de fósforo foram aplicadas, indicando um aliviamento dos efeitos adversos da compactação pela aplicação de maiores doses de fósforo (Figura 4 e Quadro 6).

À medida que o grau de compactação aumenta para a dose $100 \mathrm{mg} \mathrm{dm}^{-3}$, ocorre uma redução linear da MSPA. Nas doses 200 e $400 \mathrm{mg} \mathrm{dm}^{-3}$, as equações de regressão que correlacionam a MSPA, e PAPA com o grau de compactação, seguiram uma equação do segundo grau, sendo que na dose $400 \mathrm{mg} \mathrm{dm}^{-3}$, obteve-se a MSPA máxima $\left(5,81 \mathrm{~g} \mathrm{~kg}^{-1}\right)$ que ocorreu no $\mathrm{GC}=65 \%$ e o PAPA máximo $\left(19,61 \mathrm{mg} \mathrm{kg}^{-1}\right)$ que ocorreu no $\mathrm{GC}=65 \%$. Os menores valores de MSPA e PAPA foram obtidos onde não ocorreu a aplicação de fósforo e para o $\mathrm{GC}=95 \%$.

De acordo com os resultados obtidos verifica-se que o fósforo pode ser considerado como um fator de aliviamento da compactação do solo corroborando com os estudos feitos por alguns autores, que afirmam que apesar da compactação do solo poder causar uma concentração superficial das raízes (BARBER, 1995), a aplicação de nutrientes, como fósforo e o nitrogênio, em linhas, faixas ou a lanço, na superfície do solo, pode afetar o crescimento radicular, o estado nutricional das plantas e o desenvolvimento das culturas (ANGHINONI, 1992; ANGHINONI \& BARBER, 1980; BORKET \& BARBER, 1985; KLEPKER \& ANGHINONI, 1993, 1995), permitindo assim, obter maiores produções em condições físicas desfavoráveis por minimizar o efeito da compactação na produtividade. 


\section{LAT OSSOL O VERMELHO DISTRÓFICO TÍPIC O}

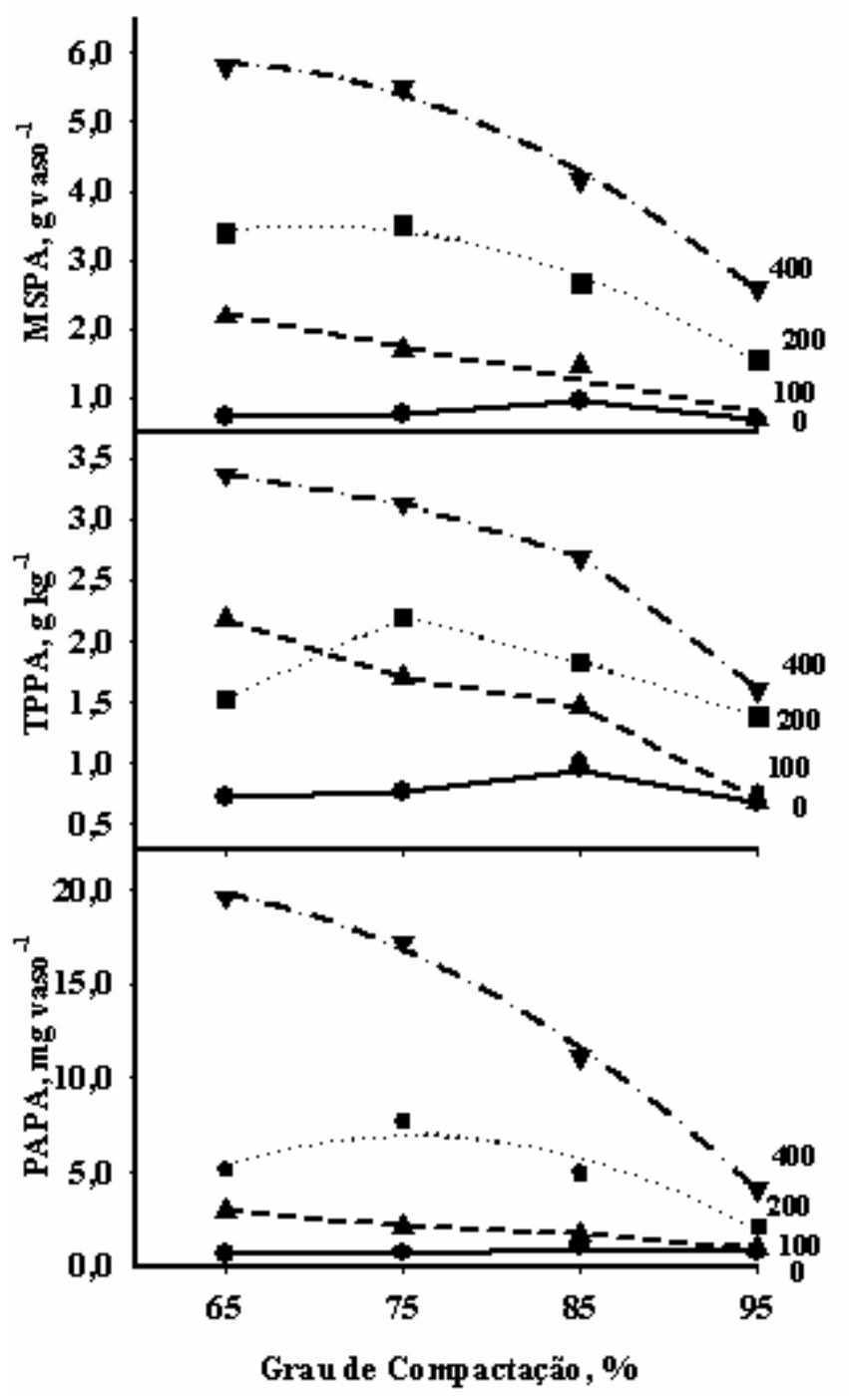

FIGURA 4 - Matéria Seca da Parte Aérea (MSPA), Teor de Fósforo na Parte Aérea (TPPA) e Fósforo Acumulado na Parte Aérea (PAPA) de plantas de milho cultivadas em um Latossolo Vermelho Distrófico típico. 
QUADRO 6 - Equações significativas ao nível de 5\% (Latossolo Vermelho distrófico):

\begin{tabular}{|c|c|c|c|c|c|c|}
\hline \multirow{2}{*}{$\begin{array}{c}\text { Grau de } \\
\text { compactação } \\
(\%)\end{array}$} & \multicolumn{4}{|c|}{$\begin{array}{c}\text { Dose de Fósforo } \\
\left(\mathrm{mg} \mathrm{dm}^{-3}\right)\end{array}$} & \multicolumn{2}{|l|}{$\begin{array}{l}\text { Equações da Regressão } \\
\text { Significancia à } 5 \%\end{array}$} \\
\hline & ( & 100 & 200 & 400 & & $\overline{\mathbf{R}^{2}}$ \\
\hline \multicolumn{7}{|c|}{ Matéria seca da Parte Aérea - MSPA $\left(\mathrm{g} \mathrm{vaso}^{-1}\right)$} \\
\hline 65 & 0,72 & 2,18 & 3,69 & 5,81 & $\mathrm{MSPA}_{(0)}=\mathrm{NS}$ & \\
\hline 75 & 0,77 & 1,70 & 3,51 & 5,50 & $\operatorname{MSPA}_{(100)}=5,270-0,047 \mathrm{GC}$ & 0,95 \\
\hline 85 & 0,95 & 1,47 & 2,67 & 4,15 & MSPA $_{(200)}=-11,5845+0,4324 \mathrm{GC}-0,003 \mathrm{GC}^{2}$ & 0,99 \\
\hline 95 & 0,68 & 0,69 & 1,55 & 2,58 & $\operatorname{MSPA}_{(400)}=-6,4243+0,3926 \mathrm{GC}-0,0032 \mathrm{GC}^{2}$ & 0,99 \\
\hline \multicolumn{7}{|c|}{ Teor de Fósforo na Pate Aérea - TPPA $\left(\mathrm{g} \mathrm{kg}^{-1}\right)$} \\
\hline 65 & 0,95 & 1,37 & 1,53 & 3,37 & $\mathrm{MSPA}_{(0)}=\mathrm{NS}$ & \\
\hline 75 & 0,97 & 1,26 & 2,20 & 3,13 & $\operatorname{MSPA}_{(100)}=\mathrm{NS}$ & \\
\hline 85 & 1,07 & 1,20 & 1,83 & 2,69 & $\operatorname{MSPA}_{(200)}=\mathrm{NS}$ & \\
\hline 95 & 1,13 & 1,39 & 1,38 & 1,60 & $\operatorname{MSPA}_{(400)}=\mathrm{NS}$ & \\
\hline \multicolumn{7}{|c|}{ Fósforo Acumulado na Parte Aérea - PAPA $\left(\mathrm{mg} \mathrm{vaso}^{-1}\right)$} \\
\hline 65 & 0,69 & 2,98 & 5,16 & 19,61 & $\mathrm{MSPA}_{(0)}=\mathrm{NS}$ & \\
\hline 75 & 0,74 & 2,16 & 7,72 & 17,22 & $\operatorname{MSPA}_{(100)}=\mathrm{NS}$ & \\
\hline 85 & 1,02 & 1,75 & 4,98 & 11,15 & $\operatorname{MSPA}_{(200)}=-70,002+2,035 \mathrm{GC}-0,013 \mathrm{GC}^{2}$ & 0,91 \\
\hline 95 & 0,77 & 0,96 & 2,16 & 4,15 & $\operatorname{MSPA}_{(400)}=-17,3269+1,3195 \mathrm{GC}-0,0115 \mathrm{GC}^{2}$ & 0,99 \\
\hline
\end{tabular}

\section{CONCLUSÕES}

As maiores produções de MSPA, TPPA e PAPA foram obtidas nos graus de compactação $72 \%$ no RQ; entre 75 e $77 \%$ no LVAd e $65 \%$ no LVd, evidenciando o efeito benéfico de um certo grau de compactação.

Em solos com um maior grau de compactação, foi necessário a aplicação de uma maior dose de fósforo para aumentar a produção, sugerindo maiores gastos com adubos em solos compactados.

Em solos compactados, o fósforo funcionou como um fator de aliviamento do efeito da compactação, resultando em uma maior produção.

\section{REFERÊNCIAS BIBLIOGRÁFICAS}

ANGHINONI, I. Uso do fósforo pelo milho afetado pela fração de solo fertilizada com fosfato solúvel. Revista Brasileira de Ciência do Solo, Viçosa, v. 16, p. 349-353, 1992.

ANGHINONI, I.; BARBER, S. A. Phosphorus influx and growth characteristcs of corn roots as influenced by phosphorus supply. Agronomy Journal, Madison, v. 72, p. 682-688, 1980.

BARBER, S. A. Soil nutrient biavailability: a mechanistic approach. New York: J. Wiley \& Sons, 1995. 414 p.

BARZEGAR, A. R.; ASSODAR, M. A. Effectiveness of sugarcane residue incorporation at different water contents and proctor compaction loads in reducing soil compactibility. Soil \& Tillage Research, Amsterdam, v. 57, p. 167-172, 2000.

BORGES, E. N. Efeito de doses de gesso + massa seca de crotalária e de níveis de compactação em atributos físicos de um latossolo vermelho-escuro. 1995. $136 \mathrm{f}$. Tese (Doutorado) - Escola Superior de Agricultura "Luiz de Queiroz”, Piracicaba, 1995.

BORGES, E. N.; LOMBARDINETO, F.; CORRÊA, G. F.E.; BORGES, E. V. S. Alterações físicas introduzidas por diferentes níveis de compactação em Latossolo Vermelho Escuro textura média. Pesquisa Agropecuária Brasileira, Brasília, v. 34, p. 1663-1667, 1999. 
BORKET, C. M.; BARBER, S. A. Soybean shooot and root growth and phosphorus concentration as affected by phosphate placement. Soil Science American Journal, Madison, v. 49, p. 152-155, 1985.

CAMARGO, O. A.; ALLEONI, L. R. F. Compactação do solo e o desenvolvimento das plantas. Piracicaba: [s.n.], 1997. $132 \mathrm{p}$.

CORRÊA, J. B. D.; ANDRADE, L. A.; DIAS JUNIOR, M. de S.; ALVES, V. G. Efeito da compactação na concentração foliar de nutrientes na cana-de-açúcar em três tipos de solos. In: FERTBIO'98, 1998, Lavras. Anais... Lavras: [s.n.], 1998. p. 57.

DIAS JUNIOR, M. de S.; ESTANISLAU, W. T. Grau de compactação e retenção de água de latossolos submetidos a diferentes sistemas de manejo. Revista Brasileira de Ciência do Solo, Campinas, v. 23, p. 45-51, 1999.

DIAS JUNIOR, M. de S.; PIERCE, F. J. O processo de compactação do solo e sua modelagem. Revista Brasileira de Ciência do Solo, Campinas, v. 20, p. 175-182, maio/ago. 1996.

DÍAZ-ZORITA, M. Effect of deep-tillage and nitrogen fertilization interactions on dryland corn (Zea mays L.) productivity. Soil \& Tillage Research, Amsterdam, v. 54, p. 11-19, 2000.

EKWUE, E. I.; STONE, R. J. Density-moisture relations of some trinidadian soils incorporated with sewage sludge. Transactions American Society Agricultural Engineers, Saint Joseph, v. 40, 1997.

EMPRESABRASILEIRADEPESQUISAAGROPECUÁRIA. Centro Nacional de Pesquisa de Solos. Manual de métodos de análise de solo. 2. ed. Rio de Janeiro, 1997. 212 p.

FIGUEIREDO, L. H. A.; DIAS JUNIOR, M. S. Efeito dos sistemas de preparo do solo na $\mathrm{Ds}_{\text {max }}$ e umidade ótima de compactação em um Latossolo Roxo. In: CONGRESSO BRASILEIRO DECIÊNCIA DO SOLO, 1997, Rio de Janeiro. Anais... Rio de Janeiro: SBCS, 1997. p. 26.

FOLONI, J. S. S.; CALONEGO, J. C.; LIMA, S. L. Efeito da compactação do solo no desenvolvimento aéreo e radicular de cultivares de milho. Pesquisa Agropecuária Brasileira, Brasília, v. 38, p. 947-955, 2003.
GRANT, C. A.; FLATEN, D. N.; TOMASIEWICZ, D. J.; SHEPARD, S. C. A importância do fósforo no desenvolvimento inicial da planta. [S.l.]: POTAFOS, 2001.

HINGSTON, F. J.; POSNER, A. M.; QUIRQ, J. P. Anion adsorption by goethite and gibbsite: I. the role of proton in determing adsorption envelopes. Journal Soil Science, Baltimore, v. 23, p. 177-192, 1972.

KLEPKER, D.; ANGHINONI, I. Phosphate uptake and corn root distribution as affected by fertilizer placement and soil tillage. Agronomy Trends in Agril. Science, [S.1.], v. 1, p. 111-115, 1993.

KLEPKER, D.; ANGHINONI, I. Crescimento radicular e aéreo do milho em vasos em função do nível de fósforo no solo e da localização do adubo. Revista Brasileira de Ciência do Solo, Campinas, v. 19, p. 403-408, 1995.

KRZIC, M.; FORTIN, M. C.; BOMKE, A. A. Short-term responses of soil physical proprieties to corn tillageplanting systems in a humid maritime climate. Soil \& Tillage Research, Amsterdam, v. 54, p. 171-178, 2000.

MIRANDA, E. E. V. Avaliação da sustentabilidade da estrutura de um Latossolo sob cultivo de cafeeiro na Região dos Cerrados. 2001. 84 p. Dissertação (Mestrado em Solos e Nutrição de Plantas) - Universidade Federal de Lavras, Lavras, 2001.

MIRANDA, E.E. V.; DIAS JUNIOR, M. S. Comportamento da curva de compactação de cinco solos da região de Lavras (MG).In:CONGRESSOBRASILEIRODECIÊNCIADOSOLO, 1998, Brasília. Resumos... Brasília: [s.n.], 1998. CD-ROM.

MORAES, M. H. Efeito da compactação em algumas propriedades físicas do solo e no desenvolvimento do sistema radicular de plantas de soja (Glycine max (L) MERRIL). 1988. 106 f. Dissertação (Mestrado) - Escola Superior de Agricultura "Luiz de Queiroz", Piracicaba, 1988.

OLIVEIRA, G. C.; DIAS JUNIOR, M. S.; RESK, D. V. S.; CURI, N. Alterações estruturais e comportamento compressivo de um Latossolo Vermelho distrófico argilososob sistemas de uso e manejo. Pesquisa Agropecuária Brasileira, Brasília, v. 38, n. 2, p. 291-299, 2003. 
PACHECO, A. A. R. C.; DIAS JUNIOR, M. S. Estudo comparativo de métodos de campo e laboratório aplicados à confecção de blocos em adobe. Ciência e Prática, Lavras, v. 14, p. 176-190, 1990.

QUEIROZ-VOLTAN, R. B.; NOGUEIRA, S. dos S. S.; MIRANDA, M. A. C. Aspectos da estrutura da raiz e do desenvolvimento de plantas de soja em solos compactados. Pesquisa Agropecuária Brasileira, Brasília, v. 35, p. 929-938, 2000.

SANTOS, G. A.; SOUZA, C. A. S.; DIAS JUNIOR, M. S.; FURTINI NETO, A. E.; GUIMARÃES, P. T. G. Disponibilidade de fósforo e produção de matéria seca de plantas de milho (Zea mays L.), cultivadas em diferentes graus de compactação e fertilização, em um Latossolo Vermelho Amarelo. In: CONGRESSO BRASILEIRO DE CIÊNCIADO SOLO, 1998, Brasília. Anais... Brasília: [s.n.], 1998. CD-ROM.

SARRUGE, J. R.; HAAG, H. P. Análises químicas em plantas. Piracicaba: ESALQ, 1974.56 p.

SAWHNEY, B. L. Charge characteristics of soils as affected by phosphate sortion. Soil Science Society of America Proceedings, Madison, v. 38, n. 1, p. 159-160, Jan./Feb. 1974.
SHIERLAW, J.; ALSTON, A. M. Effect of soil compaction on root growth an uptake of phosphorus. Plant and Soil, The Hague, v. 77, p. 15-26, 1984.

SILVA, R. B. da. Efeito da adsorção de fósforo em parâmetros físicos e na compressibilidade de solos da micro-região dos Campos da Mantiqueira, MG. 1998. 109 f. Dissertação (Mestrado) - Universidade Federal de Lavras, Lavras, 1998.

SILVA, R. B. da. Efeito da adsorção de fosfato em parâmetros físicos e na compressibilidade de solos tropicais. Revista Brasileira de Ciências do Solo, Campinas, v. 23, p. 219226, abr./jun. 1999.

SPOSITO, G. Surface reactionns in natural and aqueous colloidal systems. Chimia, Zurich, v. 43, p. 169-176, 1989.

STANCATI, G; NOGUEIRA, J. B.; VILAR, O. M. Ensaios de laboratóriodemecânica dos solos. São Carlos: USP, 1981.208p.

TORRES, E.; SARAIVA, O. F.; GALERANI, P. R. Manejo do solo para cultura da soja. Londrina: EMBRAPA-CNPSo, $1993.71 \mathrm{p}$.

VEPRASKAS, M. J. Plant response mechanisms to soil compaction. In: WILKINSON, R. E. Plant-environment interactions. [S.1.: s.n.], 1994. p. 263-287. 